\title{
A 12-year-old girl with absent radial pulse: arterial thoracic outlet syndrome with subclavian artery aneurysm and thrombosis of the brachial artery
}

\author{
Schroeder, S ; Cannizzaro, E ; Kellenberger, C J ; Saurenmann, R K
}

\begin{abstract}
Brachial arterial occlusion is rare in children and adolescents. Once a traumatic cause is excluded, the differential diagnosis consists of a variety of rare conditions. We report the case of a 12year-old girl whose presenting symptoms-an absent radial pulse and Raynaud's phenomenon of the right hand-could be easily mistaken for a vasculitis. She was found to have arterial thoracic outlet syndrome with right subclavian artery compression and aneurysm formation caused by an anomalous first rib and consecutive thromboembolic occlusion of the brachial artery. The diagnosis and differential diagnosis of this condition are reviewed.
\end{abstract}

DOI: https://doi.org/10.1007/s00431-012-1748-y

Posted at the Zurich Open Repository and Archive, University of Zurich

ZORA URL: https://doi.org/10.5167/uzh-74238

Journal Article

Published Version

Originally published at:

Schroeder, S; Cannizzaro, E; Kellenberger, C J; Saurenmann, R K (2012). A 12-year-old girl with absent radial pulse: arterial thoracic outlet syndrome with subclavian artery aneurysm and thrombosis of the brachial artery. European Journal of Pediatrics, 171(11):1707-1709.

DOI: https://doi.org/10.1007/s00431-012-1748-y 


\title{
A 12-year-old girl with absent radial pulse: arterial thoracic outlet syndrome with subclavian artery aneurysm and thrombosis of the brachial artery
}

\author{
S. Schroeder • E. Cannizzaro • C. J. Kellenberger • \\ R. K. Saurenmann
}

Received: 6 March 2012 /Accepted: 12 April 2012 /Published online: 28 April 2012

(C) Springer-Verlag 2012

\begin{abstract}
Brachial arterial occlusion is rare in children and adolescents. Once a traumatic cause is excluded, the differential diagnosis consists of a variety of rare conditions. We report the case of a 12-year-old girl whose presenting symptoms - an absent radial pulse and Raynaud's phenomenon of the right hand - could be easily mistaken for a vasculitis. She was found to have arterial thoracic outlet syndrome with right subclavian artery compression and aneurysm formation caused by an anomalous first rib and consecutive thromboembolic occlusion of the brachial artery. The diagnosis and differential diagnosis of this condition are reviewed.
\end{abstract}

Keywords Absent pulse · Raynaud's phenomenon · Aneurysm $\cdot$ Vasculopathy $\cdot$ Thoracic outlet syndrome

\section{Case report}

A previously healthy 12-year-old girl was referred to our hand surgery department with a 3-week history of right arm pain accompanied by finger discoloration and paraesthesia. No trauma or other precipitating event was retrievable on history. On physical examination, the right radial artery pulse was undetectable and the systolic blood pressure was $20 \mathrm{mmHg}$ lower than on the left arm while sensitivity, muscle strength and reflexes were normal. Magnetic resonance imaging including

S. Schroeder $(\bowtie) \cdot$ E. Cannizzaro $\cdot$ R. K. Saurenmann

Rheumatology, University Children's Hospital,

Steinwiesstr. 75,

8032 Zurich, Switzerland

e-mail: silke.schroeder@kispi.uzh.ch

C. J. Kellenberger

Diagnostic Imaging, University Children's Hospital,

Zurich, Switzerland contrast-enhanced angiography (MRA) revealed a fusiform aneurysm of the subclavian artery with wall irregularities and a stenosis of the brachial artery. Vasculitis was suspected and the girl was referred to the paediatric rheumatology clinic. A work-up for the suspected diagnosis of Takayasu arteritis showed normal inflammation parameters. MRA of the chest and the abdomen revealed a completely normal thoracic and abdominal aorta including its major branches except for the right subclavian and brachial arteries. After an interval with spontaneous improvement of symptoms for 2 months, she returned to a follow-up appointment reporting an increased weakness in her right arm. Follow-up MRA revealed disease progression with now partial thrombosis of the subclavian artery aneurysm and complete occlusion of the brachial artery (Fig. 1). Again no laboratory signs of inflammation were detectable. Anticoagulation therapy with low molecular weight heparin was initiated.

Additional differential diagnoses were evaluated such as coagulopathies, fibromuscular dysplasia and lipid metabolic disorders. Echocardiography and ECG were both normal. Extensive blood work showed normal coagulation parameters and hemostasis activator and inhibitor parameters, and no autoantibodies or other pathologic findings were detectable. Finally, detection of bilateral rib anomalies on a chest radiograph, with bridging and pseudoarticulation of the first and second ribs, led to the tentative diagnosis of an arterial thoracic outlet syndrome (TOS). The diagnosis was confirmed by contrast-enhanced computed tomography (CT) performed with different arm positions showing compression of the right subclavian artery between the clavicle and the bridging ribs (Fig. 2). CT also revealed recanalisation of the right brachial artery within 1 month after the start of the heparin therapy.

A resection of the first rib and of the subclavian aneurysm was performed 1 year after the initial presentation. The right 


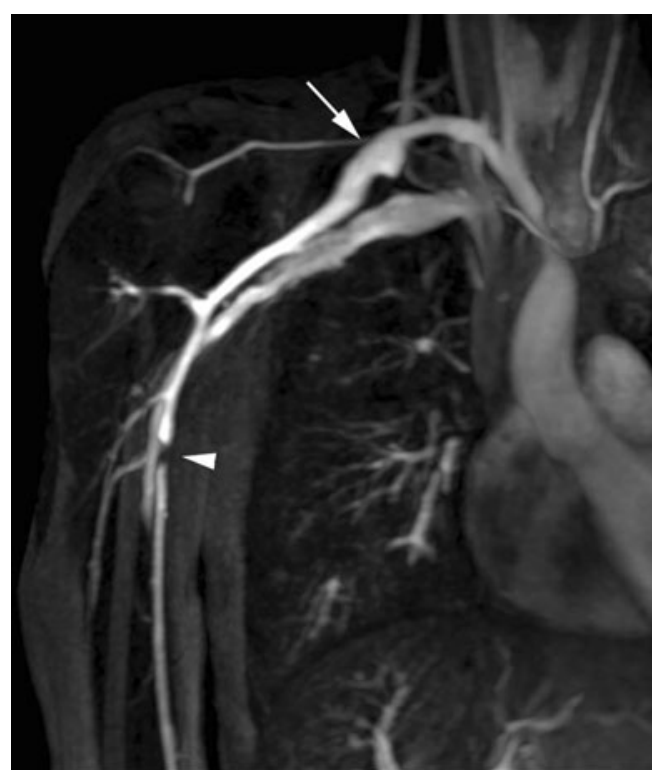

Fig. 1 Coronal MRA image shows aneurysm of the right subclavian artery (arrow) with peripheral filling defect due to a marginal thrombus and localised occlusion of the brachial artery (arrowhead)
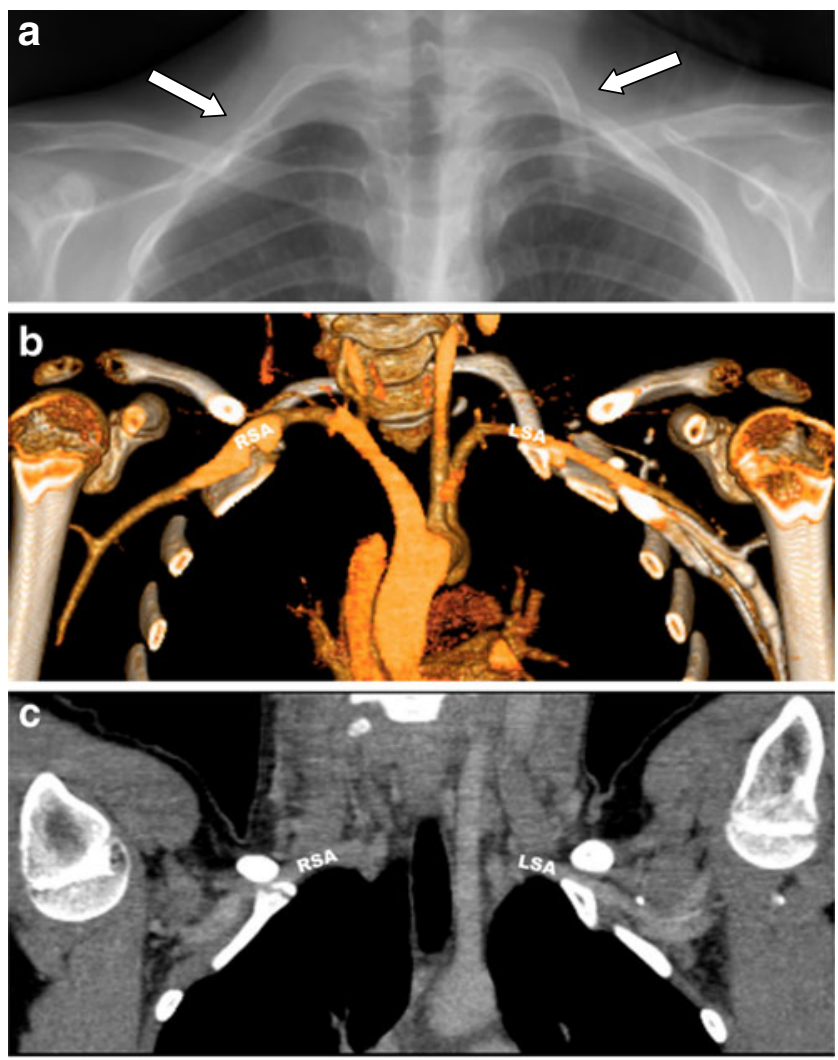

Fig. 2 a The common chest X-ray, which demonstrates bridging of the first and second ribs with pseudoarticulation bilaterally and the coronal contrast-enhanced CT images obtained with the arms positioned by the sides (b) and elevated (c). b Volume-rendered image shows the aneurysm of the right subclavian artery $(R S A)$ and a normal left subclavian artery $(L S A)$ at the thoracic outlet. c With the arms elevated, there is severe narrowing of the right subclavian artery $(R S A)$ caused by compression between the clavicle and rib anomaly radial artery pulse normalised immediately and circulation remained normal ever after. However, the postoperative course was complicated by a paralysis of the right lower plexus, which only gradually resolved and required 4 months of intensive physiotherapy and occupational therapy. Currently our patient is doing well without sequelae.

\section{Discussion}

The presentation of an adolescent girl with a missing radial pulse and proximal artery wall irregularities prompted us to suspect Takayasu arteritis as the first differential diagnosis in this case. The American College of Rheumatology 1990 diagnostic criteria were met with five out of six criteria being fulfilled, although the absence of inflammatory signs and the restriction to one single affected artery site were unexpected findings for this diagnosis. Only the further investigations prompted by the disease progression led to the diagnosis of a mechanical instead of an inflammatory origin of the artery occlusion.

Thoracic outlet syndrome is a condition caused by the compression of neural and/or vascular structures passing between the clavicle and the scalene muscles. The brachial plexus (neurogenic TOS), the subclavian vein (venous TOS), the subclavian artery (arterial TOS) or a combination of these can be affected in this syndrome. In children TOS is extremely rare.

Thoracic outlet syndrome is well known in adult patients; most of them present with neurologic symptoms (95\%) [1, 6, 10] usually consisting in pain, paraesthesia, numbness and weakness in the affected arm caused by the compression of the brachial plexus. Rarer forms of TOS involve the subclavian vein or subclavian artery. Signs of subclavian vein involvement are thrombosis, oedema and cyanosis of the involved extremity, whereas compression of the subclavian artery leads to palor, pulselessness and decreased temperature of the involved extremity [8]. Common causes of compression, especially of the blood vessels, are a cervical rib, a malformation of the first rib, or congenital fibromuscular bands of a tight scalene anterior muscle [3]. TOS is extremely rare in children, and similar to adults also, most paediatric cases present with neurologic symptoms [1]. The first case report of a child with vascular involvement was published by DiFiore et al. [4]. Involvement of the subclavian artery is indeed very uncommon but is associated with the highest risks of complications such as post-stenotic dilatation and aneurysm formation with partial or complete thrombosis. This can lead to distal embolisation, a complication described in adults [2]. To our knowledge, our patient is the first child presenting with embolisation into the brachial artery from a partially thrombosed subclavian artery aneurysm due to TOS. Interestingly, the rib anomaly was present on both sides in our patient but caused arterial compression on the right side only. 
In children, symptoms can initially be mild because of good arterial collaterals. In advanced stages, ischemic events such as ulceration of the digits, absent pulse or Raynaud's phenomenon may occur [9]. The extremity can appear pale and cool and blood pressure will be reduced as compared with the other side. The confirmation of a diagnosis of TOS is made by diagnostic imaging. A chest radiograph may reveal a cervical rib (56-67\% of vascular TOS) or an abnormal first rib (14-27\% of vascular TOS) [5]. Contrast-enhanced CT or MRA can show an aneurysm and, when performed with the arms elevated, may reveal compression of the subclavian artery.

Treatment of TOS can be either conservative with physiotherapy or surgical intervention with excision of the compressing rib. In case of vascular involvement and complications such as stenosis, thrombosis or aneurysm formation as well as in case of severe and persistent irritation of the plexus, a surgical intervention is indicated [7]. In our patient, surgical removal of the malformed first right rib was performed. Interestingly, Shepard et al. reported a case with complete resolution of an arterial aneurysm after a scalenectomy and excision of the malformed first rib without reconstruction of the affected vessel [11].

\section{References}

1. Arthur LG, Teich S, Hogan M, Caniano D, Smead W (2008) Pediatric thoracic outlet syndrome: a disorder with serious vascular complications. Pediatr Surg 43(6):1089-1094

2. Bearn P, Patel J, O'Fllyn WR (1993) Cervical rib: a cause of distal and cervical embolism. Postgrad Med J 69:65-68

3. Davidovic LB, Kostic DM, Jakovljevic NS et al (2003) Vascular thoracic outlet syndrome. World J Surg 27:545-550

4. Difiore JW, Reid JR, Drummond-Webb J (2002) Thoracic outlet syndrome in a child-transaxillary resection of anomalous first rib. $\mathrm{J}$ Pediatr Surg 37(8):1220-1222

5. Durham JR, Yao JST, Pearce WH et al (1995) Arterial injuries in the thoracic outlet syndrome. J Vasc Surg 21:57-70

6. Hempel GK, Shutze WP, Anderson JF et al (1996) 770 consecutive supraclavicular first rib resections for thoracic outlet syndrome. Ann Vasc Surg 10:456-463

7. Huang J, Zager E (2004) Thoracic outlet syndrome. Neurosurgery 55:897-903

8. Kremer RM, Ahlquist RE Jr (1975) Thoracic outlet compression syndrome. Am J Surg 130:612-616

9. Mackinnon SE, Novak CB (2002) Thoracic outlet syndrome. Curr Probl Surg 39:1070-1145

10. Sanders RJ (1996) Results of the surgical treatment for thoracic outlet syndrome. Semin Thorac Cardiovasc Surg 8(2):221-228

11. Shepard AD, Parikshak M (2004) Current surgical therapy. In: Cameron J (ed) Thoracic outlet syndrome. Elsevier and Mosby, Philadelphia, pp 840-845 